\title{
Validation of eight genetic risk factors in East Asian populations replicated the association of BRAP with coronary artery disease
}

\author{
Kunihiko Hinohara $^{1,2}$, Hitoshi Ohtani ${ }^{1,2}$, Toshiaki Nakajima ${ }^{1,2}$, Taishi Sasaoka ${ }^{3}$, Motoji Sawabe ${ }^{4}$, Bok-Soo Lee ${ }^{5}$, \\ Jimin $\mathrm{Ban}^{5}$, Jeong-Euy Park ${ }^{5}$, Tohru Izumi ${ }^{3}$ and Akinori Kimura ${ }^{1,2}$ \\ Coronary artery disease (CAD) is caused by a thrombotic occlusion or spasm of the coronary artery. Association of genetic \\ variants with susceptibility to CAD has been reported in various populations, but the association should be replicated in other \\ populations to establish the role of genetic variants in CAD. We conducted a case-control study with a total of 1480 CAD cases \\ and 2115 controls from two East Asian populations, Japanese and Korean, to validate the associations of CAD with eight single \\ nucleotide polymorphisms (SNPs) in eight loci, which were identified from large-scale whole-genome association studies in \\ Europeans or East Asians. Among the tested SNPs, one SNP in BRAP (rs1 1066001) showed a significant association in allele \\ frequency distribution with CAD in both the Japanese (Odds ratio (OR) $=1.63,95 \%$ confidence interval $(\mathrm{Cl}) ; 1.41-1.89$, \\ $P=5.0 \times 10^{-11}$, corrected $\left.P(P C)=4.0 \times 10^{-10}\right)$ and Korean populations $\left(\mathrm{OR}=1.68,95 \% \mathrm{Cl} ; 1.41-2.00, P=6.5 \times 10^{-9}\right.$, \\ $\left.P \mathrm{c}=5.2 \times 10^{-9}\right)$, and a meta-analysis showed a significant association in the East Asian populations $(0 \mathrm{R}=1.65,95 \% \mathrm{Cl}$; \\ 1.48-1.85, $P=1.8 \times 10^{-18}, P c=1.4 \times 10^{-17}$ ), whereas no evidence of association was found for the other SNPs. In addition, \\ a combined analysis of BRAP and another CAD locus on 9p21 suggested that these loci had a synergistic role in the \\ susceptibility. Failure to replicate the association with the other SNPs, which were reported in the European populations, \\ suggested that their contributions to CAD were not large enough to be readily captured in the East Asian populations. \\ Journal of Human Genetics (2009) 54, 642-646; doi:10.1038/jhg.2009.87; published online 28 August 2009
}

Keywords: BRAP; case-control study; coronary heart disease; SNP; validation

\section{INTRODUCTION}

Coronary artery disease (CAD) caused by a thrombotic occlusion or spasm of the coronary artery becomes a major health problem in many countries. ${ }^{1} \mathrm{CAD}$ is mainly based on coronary atherosclerosis and often manifests with sudden chest pain due to reversible (angina pectoris, AP) or irreversible (myocardial infarction, MI) ischemia in the heart caused by decreased blood flow in the coronary arteries. Although environmental or life-style factors, such as smoking, hypertension, hypercholesterolemia and diabetes mellitus, contribute to the development of $\mathrm{CAD},{ }^{2}$ genetic factors are also involved in the pathogenesis of $\mathrm{CAD}^{3}$ Several large-scale association studies using a large number of genetic variations, including single nucleotide polymorphisms (SNPs), have recently identified the susceptibility genes and loci for CAD. ${ }^{4-12}$ However, not all of the reported associations could be replicated in other studies even if middle- to large-sized samples were investigated in the original reports, suggesting that the contribution of genetic factors was not large enough to be replicated in some cases. ${ }^{13}$ Validation studies for the association in other populations are, therefore, crucial to establish the role of diseaserelated genes.

Recent progress based on the large-scale association studies has accumulated information on the susceptibility genes linked to CAD. It is noted that seven chromosomal loci were reported to be strongly associated with CAD in Europeans conducted by the Wellcome Trust Case Control Consortium (WTCCC) ${ }^{6}$ (loci on chromosomes 1q43, 5q21,6q25, 9p21, 16q23, 19q12 and 22q12). Samani et al. ${ }^{7}$ tested several loci in the WTCCC study, which met their predefined criterion $\left(P<1.2 \times 10^{-5}\right.$ and less than a $50 \%$ chance of being false-positive), in German MI families and found that three loci on chromosomes 2q36, 6 q25 and 9p21 were significantly associated with MI. In addition, a combined analysis of data from these two studies revealed four additional CAD-associated loci on chromosomes 1p13, 1q41, 10q11 and $15 q 22 .{ }^{7}$ In a recent study, three other loci were reported to be associated with early-onset $\mathrm{MI}^{10}$ (loci on chromosomes 2q33, 6q24

${ }^{1}$ Department of Molecular Pathogenesis, Medical Research Institute, Tokyo Medical and Dental University, Tokyo, Japan; ${ }^{2}$ Laboratory of Genome Diversity, School of Biomedical Science, Tokyo Medical and Dental University, Tokyo, Japan; ${ }^{3}$ Department of Internal Medicine and Cardiology, Kitasato University School of Medicine, Sagamihara, Japan; ${ }^{4}$ Department of Pathology, Tokyo Metropolitan Geriatric Medical Center, Tokyo, Japan and ${ }^{5}$ Division of Cardiology, Samsung Medical Center, Sungkyunkwan University School of Medicine, Seoul, Korea

Correspondence: Professor A Kimura, Department of Molecular Pathogenesis, Medical Research Institute, Tokyo Medical and Dental University, 1-5-45 Yushima, Bunkyo-ku, Tokyo 113-8510, Japan.

E-mail: akitis@mri.tmd.ac.jp

Received 30 June 2009; revised 10 August 2009; accepted 11 August 2009; published online 28 August 2009 
and 21q22). In addition, Gudbjartsson et al. ${ }^{11}$ reported that an SNP on chromosome 12q24 was significantly associated with MI. All these studies were conducted in European populations. On the other hand, Ozaki et al. ${ }^{12}$ identified the BRCA1-associated protein $(B R A P)$ gene as a possible binding partner of galectin-2.As they reported the gene for galectin-2 LGALS2 as a susceptibility gene for MI, ${ }^{14}$ they investigated a possible association of MI with BRAP and found an SNP (rs11066001) to be significantly associated with MI in both the Japanese and Taiwanese populations. ${ }^{12}$

We conducted validation studies in the Japanese and Korean populations, which confirmed the association of CAD with a 9p21 SNP (rs1333049), but failed to replicate the association with SNPs in LTA, LGALS2 and PSMA6, showing that not all of the associations reported in the Japanese population ${ }^{4,14}$ could be readily replicated in our studies. ${ }^{15-17}$ In this study, we investigated the association between CAD and eight SNPs in eight genome regions in the East Asian populations.

\section{MATERIALS AND METHODS}

\section{Subjects}

A total of $1480 \mathrm{CAD}$ cases and 2115 control subjects from the Japanese and Korean populations were recruited as reported previously. ${ }^{15-17}$ Briefly, the Japanese panels were composed of CAD cases $(n=622)$ and controls $(n=1402)$. The Japanese controls included healthy individuals selected at random $(n=656)$ and healthy-donor-derived B-cell lines obtained from the Japan Health Sciences Foundation $(n=746)$. The Korean subjects consisted of CAD cases $(n=858)$ and controls $(n=713)$. The Korean controls included healthy individuals selected at random $(n=179)$ and cancer patients without CAD $(n=534)$ consisting of 230,147, 87 and 71 patients with stomach cancer, colon cancer, lung cancer and hepatocellular carcinoma, respectively. The diagnosis of CAD was based on the standard criteria as described previously. ${ }^{15-17}$ In brief, the diagnosis of MI was based on typical electrocardiographic changes, increased serum levels of creatinine kinase, aspartate aminotransferase, and lactate dehydrogenase and increased serum concentration of troponin $\mathrm{T}$. The diagnosis was confirmed by the presence of a wall motion abnormality on left ventriculography and by the identification of responsible stenosis in the coronary arteries on coronary angiography. On the other hand, AP was diagnosed by the clinical manifestation of sudden chest pain, transient electrocardiographic changes, and significant stenosis of the coronary arteries found in coronary angiography. Severity of coronary atherosclerosis was classified according to the number of coronary vessels with significant stenosis (angiographic luminal stenosis $>50 \%$ ) as $0-$, 1-, 2- or 3-vessel disease. Informed consent was received from each participant and the study was approved by the Ethics Review Boards of the Medical Research Institute of Tokyo Medical and Dental University, Kitasato University School of Medicine, Tokyo Metropolitan Geriatric Medical Center, and Samsung Medical Center.

\section{Genotyping}

Eight SNPs were selected for the validation study, because they were reported to have a minor allele frequency over 0.05 in the Asian populations (Japanese and Han Chinese) in the HapMap data (Supplementary Table 1). All SNPs were genotyped using the TaqMan SNP genotyping assay (Applied Biosystems, Foster City, CA, USA).

\section{Statistical analysis}

A statistical analysis for power and sample size computations of case-control design was performed using the PGA program (http://dceg.cancer.gov/bb/tools/ pga). ${ }^{18}$ Frequencies of alleles or genotypes were compared between the cases and controls using a $\chi^{2}$-test. The strength of the association was expressed by OR. Meta-analysis was performed using a Mantel-Haenszel method to investigate the association in the combined Japanese and Korean populations. The significance of the association between the severity of coronary atherosclerosis and rs11066001 was examined by using the Mann-Whitney $U$-test.

A stratification analysis was carried out according to the method described by Svejgaard and Ryder ${ }^{19}$ to test the independency or synergistic effects of two different alleles that were significantly associated with the disease. Briefly, basic data for the analysis were the entries of the two-by-four table giving the four phenotypic combinations of factors A and B in cases and controls, and the data were analyzed in two-by-two tables involving the stratification of each of the two factors against the other. In the analysis, contributions of factors $\mathrm{A}$ and $\mathrm{B}$ could be evaluated as odds ratios (OR) conferred by one of the factors $\left(\mathrm{OR}_{\mathrm{A}}\right.$, non- $\mathrm{B}$, or $\left.\mathrm{OR}_{\text {non- } \mathrm{A}, \mathrm{B}}\right)$ or both factors $\left(\mathrm{OR}_{\mathrm{A}, \mathrm{B}}\right)$ vs non- $\mathrm{A}$ non- $\mathrm{B}$. If these factors independently conferred risk of disease, $O R_{A, \text { non- } B}$ and $O R_{\text {non- } A, B}$ were equal to (or at least not exceeding) $\mathrm{OR}_{\mathrm{A}, \mathrm{B}}$. In contrast, when $\mathrm{OR}_{\mathrm{A}, \mathrm{B}}$ was larger than $\mathrm{OR}_{\mathrm{A}, \text { non- } \mathrm{B}}$ or $\mathrm{OR}_{\text {non- } \mathrm{A}, \mathrm{B}}$, these factors were considered to synergistically contribute to the risk.

Corrected $P(P \mathrm{c})$ values were obtained by multiplying the $P$-values with the number of independent tests (eight for the replication study and eight for the stratification analysis). When the Pc value was less than 0.05 , the association was considered to be significant.

\section{RESULTS}

Demographic characteristics of the study population are listed in Table 1. This study was conducted to replicate the association of CAD with recently reported genetic risk variants on chromosomes 1p13, $1 \mathrm{q} 41,1 \mathrm{q} 43,2 \mathrm{q} 36,5 \mathrm{q} 21,10 \mathrm{q} 11$ and $16 \mathrm{q} 23$. An SNP in BRAP on chromosome 12q24 was also tested for the association. Statistical power analysis of our study design to verify whether it could provide adequate powers in replicating the association with the SNPs on chromosomes 1p13, 1q41, 1q43, 2q36, 5q21, 10q11, 16q23 and 12q24 showed that the statistical powers were 93.3, 99.5, 99.8, 86.1, 99.9, $100.0,99.2$ and $100.0 \%$, respectively, when the Japanese and Korean populations were combined, assuming that they were genetically identical. On the other hand, the powers to detect the association either in the Japanese or Korean populations were 88.6, 98.5, 99.0, 79.7, 99.6, 100.0, 97.7 and $100.0 \%$, respectively, when they were assumed to be genetically different (Supplementary Table 2).

Genotype distributions for all SNPs were in the Hardy-Weinberg equilibrium in the tested populations (data not shown). As shown in Table 2, the SNP in BRAP showed a significant association with $\mathrm{CAD}$ in allele distribution in both the Japanese $(\mathrm{OR}=1.63,95 \% \mathrm{CI}$; $\left.1.41-1.89, P=5.0 \times 10^{-11}, P \mathrm{c}=4.0 \times 10^{-10}\right)$ and Korean populations $\left(\mathrm{OR}=1.68,95 \% \mathrm{CI} ; 1.41-2.00, P=6.5 \times 10^{-9}, P \mathrm{c}=5.2 \times 10^{-8}\right)$. When we performed a meta-analysis of data from the Japanese and Korean populations, the association with $\mathrm{CAD}$ was highly significant $\left(P=1.8 \times 10^{-18}, P c=1.4 \times 10^{-17}\right)$ with an OR of $1.65(95 \% \mathrm{CI}=1.48$ $1.85)$ for the risk allele. In addition, when the CAD patients were grouped into AP and MI, the meta-analysis showed that the

Table 1 Clinical characteristics of the study population

\begin{tabular}{|c|c|c|c|c|}
\hline & \multicolumn{2}{|c|}{ Japanese Population } & \multicolumn{2}{|c|}{ Korean Population } \\
\hline & $\begin{array}{c}C A D \\
(\mathrm{n}=622)\end{array}$ & $\begin{array}{c}\text { Control } \\
(n=1402)\end{array}$ & $\begin{array}{c}C A D \\
(\mathrm{n}=858)\end{array}$ & $\begin{array}{c}\text { Control } \\
(\mathrm{n}=713)\end{array}$ \\
\hline Age (years) & $59.3 \pm 10.2$ & $39.0 \pm 10.6$ & $61.2 \pm 11.1$ & $58.4 \pm 11.5$ \\
\hline BMI & $23.7 \pm 2.9$ & NA & $24.7 \pm 2.7$ & NA \\
\hline Gender ( $\%$ male) & 83.2 & 56.3 & 76.4 & 64.8 \\
\hline Smoking (\%) & 73.8 & NA & 69.3 & NA \\
\hline $\mathrm{HT}(\%)$ & 53.1 & NA & 45.1 & NA \\
\hline $\mathrm{HC}(\%)$ & 49.9 & NA & 43.7 & NA \\
\hline DM (\%) & 30.5 & NA & 29.1 & NA \\
\hline
\end{tabular}

Characteristics of panels used in this study are shown. The values are means \pm s.d. and percentages where indicated. Smoking: current or former smoking of $\geqslant 10$ cigarettes daily. HT: hypertension, systolic blood pressure of $\geqslant 140 \mathrm{~mm} \mathrm{Hg}$, diastolic blood pressure of $\geqslant 90 \mathrm{~mm} \mathrm{Hg}$, or taking antihypertensive medication. $\mathrm{HC}$ : hypercholesterolemia, serum total cholesterol of $\geqslant 5.72 \mathrm{mmol}^{-1}(220 \mathrm{mg}$ per $100 \mathrm{ml})$ or taking lipid-lowering medication. DM: diabetes mellitus, fasting blood glucose of $\geqslant 6.93 \mathrm{mmoll}^{-1}$ ( $126 \mathrm{mg}$ per $\left.100 \mathrm{ml}\right)$, glycosylated hemoglobin of $\geqslant 6.5 \%$, or taking anti-diabetes medication. NA, data not available. 
Table 2 Validation of eight genetic risk variants for the association with CAD

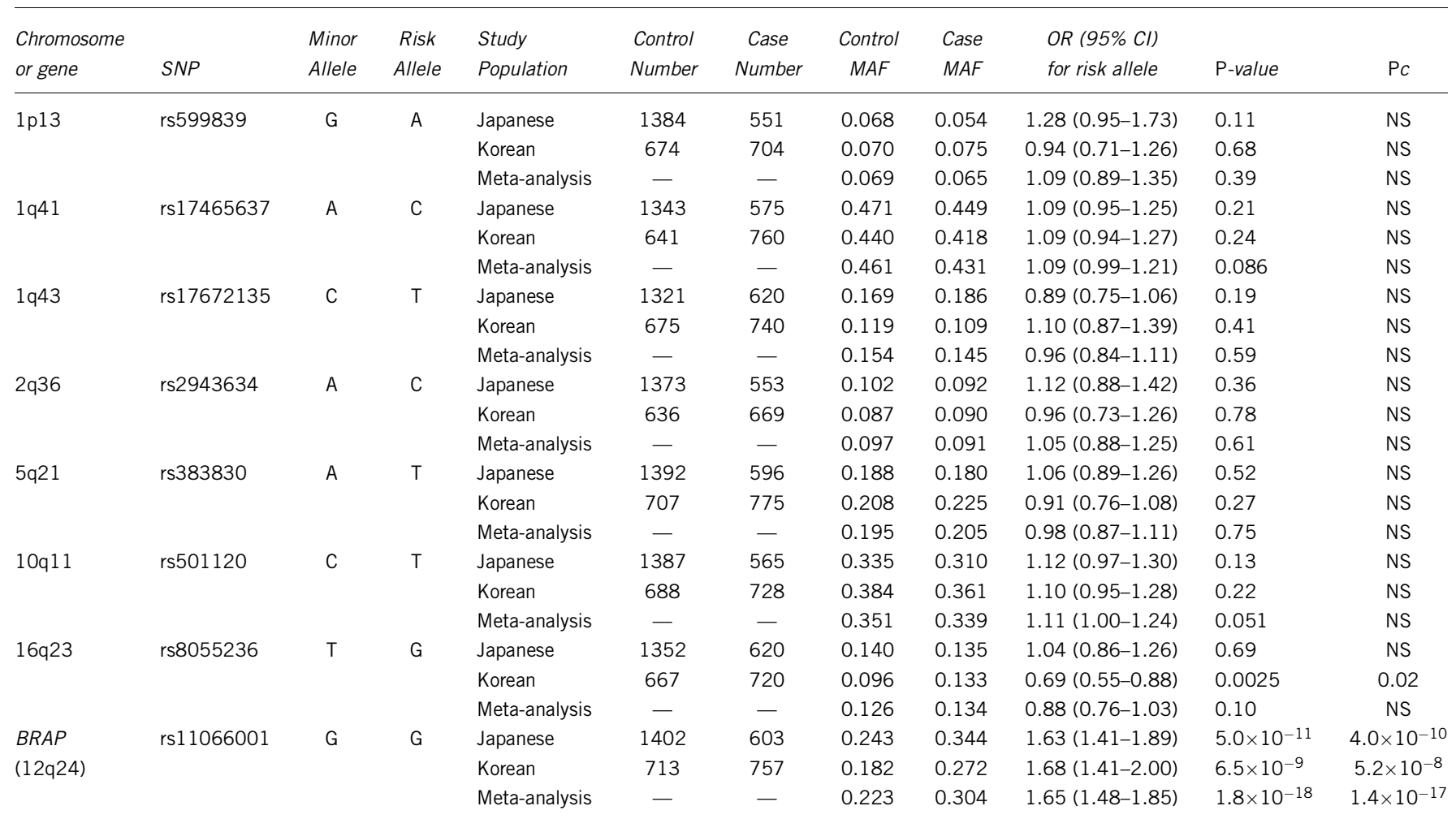

Abbreviations: $\mathrm{CAD}$, coronary artery disease; MAF, minor allele frequency; $\mathrm{OR}$, odds ratio; $\mathrm{Cl}$, confidence interval; $P \mathrm{c}, P$-value after Bonferroni's correction; NS, not significant ( $P>0.05)$. For the meta-analysis of combined Japanese and Korean groups, OR and $P$-value were calculated using a Mantel-Haenszel model. Numbers of controls and cases were not indicated for the metaanalysis because they were indicated separately in the Japanese and Korean populations.

association was significant in both groups; $\mathrm{OR}=1.49,95 \% \mathrm{CI}=1.22$ $1.82, P_{\mathrm{c}}=7.0 \times 10^{-5}$ for $\mathrm{AP}(n=369)$ and $\mathrm{OR}=1.71,95 \% \mathrm{CI}=1.52-$ $1.93, P_{\mathrm{c}}=1.4 \times 10^{-19}$ for MI $(n=1360)$. To investigate the mode of inheritance, we calculated genotype-specific ORs for rs11066001 in $B R A P$, and the results showed that ORs for AA, AG and GG genotypes were $0.55,1.52$ and 2.04 , respectively, suggesting the additive model (Supplementary Table 3).

The association with the SNP in BRAP was investigated in relation to the presence of classical risk factors including gender, age, smoking, hypertension, hypercholesterolemia, obesity and diabetes mellitus, and the severity of coronary atherosclerosis (number of vessels with significant luminal stenosis) in patients whose coronary angiographic data were available (598 Japanese cases and 453 Korean cases). It was observed that the association was not depending on the classical risk factors, suggesting that the BRAP SNP was an independent risk factor for $\mathrm{CAD}$ (data not shown). On the other hand, the $\mathrm{G}$ allele frequencies of rs11066001 in the Japanese CAD patients with $0,1,2$ and 3 vessel disease were 0.36 (vs 0.24 in the Japanese controls, $n=18$, $\mathrm{OR}=1.76, \quad P \mathrm{c}=\mathrm{ns}), \quad 0.32 \quad\left(n=247, \quad \mathrm{OR}=1.49, \quad P \mathrm{c}=1.2 \times 10^{-3}\right), \quad 0.34$ $\left(n=163, \mathrm{OR}=1.63, P \mathrm{c}=6.8 \times 10^{-4}\right)$, and $0.37 \quad(n=170, \mathrm{OR}=1.83$, $\left.P c=3.1 \times 10^{-6}\right)$, respectively, showing that the association was relatively strong in patients with severe CAD but the trend was not statistically significant (Mann-Whitney's $U$-test, $P=\mathrm{ns}$ ). In contrast, the data in the Korean CAD patients were 0.18 (vs 0.18 in the Korean controls, $n=11, \mathrm{OR}=1.00, P \mathrm{c}=\mathrm{ns}), 0.26(n=210, \mathrm{OR}=1.58$, $\left.P \mathrm{c}=3.6 \times 10^{-3}\right), \quad 0.34 \quad\left(n=148, \mathrm{OR}=2.33, P \mathrm{c}=6.4 \times 10^{-9}\right)$ and 0.35 $\left(n=84, \mathrm{OR}=2.38, P \mathrm{c}=4.0 \times 10^{-6}\right)$, respectively, showing a significant correlation between the G-allele frequency and the severity of coronary atherosclerosis (Mann-Whitney's $U$-test, $P=5.0 \times 10^{-3}$ ). On the other hand, no significant association for East Asians was found with the other seven SNPs (Table 2), even when the patients were stratified by gender, onset age and the presence of classical risk factors (data not shown).

As we have previously reported the association of 9p21 SNP with CAD in the Japanese and Korean populations, ${ }^{15}$ it was interesting to know whether there was an interaction between 9p21 and BRAP SNP in susceptibility to CAD. We stratified the cases and controls that were genotyped for both $9 \mathrm{p} 21$ and BRAP SNPs by the presence or absence of risk genotypes, GG genotype of rs11066001 (BRAP) and CC genotype of rs1333049 (9p21) (Table 3). Separate analyses in the Japanese and Korean populations did not give conclusive results when the $P$-values were corrected for multiple tests, presumably because the numbers of samples were not large enough. However, the meta-analysis revealed significant associations for BRAP (test $\{1\}$, $\mathrm{OR}_{\mathrm{A}}=2.01, \quad P \mathrm{c}=1.3 \times 10^{-5}$ ) and $9 \mathrm{p} 21 \quad$ (test $\{2\}, \quad \mathrm{OR}_{\mathrm{B}}=1.42$, $\left.P \mathrm{c}=9.6 \times 10^{-4}\right)$ and the BRAP genotype increased the risk in both the presence and absence of the $9 \mathrm{p} 21$ genotype (test $\{3\}, \mathrm{OR}=2.31$, $P \mathrm{c}=0.018$ and test $\{4\}, \mathrm{OR}_{\mathrm{A}, \text { non- } \mathrm{B}}=1.90, P_{\mathrm{c}}=1.3 \times 10^{-3}$, respectively). In addition, contribution of the $9 \mathrm{p} 21$ genotype was significant in the absence of $B R A P$ genotype (test $\{6\}, \mathrm{OR}_{\text {non- } A, B}=1.39, P c=3.4 \times 10^{-3}$ ). The OR in the presence of both the genotypes (test $\{8\}, \mathrm{OR}_{\mathrm{A}, \mathrm{B}}=3.33$, $\left.P \mathrm{c}=2.4 \times 10^{-4}\right)$ was higher than those in the presence of one genotype (tests $\{4\}$ and $\{6\}$ ), implying a synergistic contribution of these two genetic risk factors.

\section{DISCUSSION}

Recent genome-wide association studies and candidate gene approaches have identified several susceptibility loci and genes for CAD. 
Table 3 Stratification analyses of disease-associated genetic factors in the susceptibility to CAD

\begin{tabular}{|c|c|c|c|}
\hline $\begin{array}{l}\text { rs11066001 (BRAP) } \\
\text { GG genotype }\end{array}$ & $\begin{array}{l}\text { rs1333049 (9p21) } \\
\text { CC genotype }\end{array}$ & $\begin{array}{l}\text { Patients (\%) } \\
(\mathrm{n}=586)\end{array}$ & $\begin{array}{c}\text { Controls (\%) } \\
(\mathrm{n}=625)\end{array}$ \\
\hline Presence (+) & Absence (-) & $45(7.7)$ & $35(5.6)$ \\
\hline Absence (-) & presence $(+)$ & $153(26.1)$ & $124(19.8)$ \\
\hline Absence (-) & Absence (-) & $367(62.6)$ & $456(73.0)$ \\
\hline rs11066001 (BRAP) & $r s 1333049(9 p 21)$ & Patients (\%) & Controls (\%) \\
\hline GG genotype & CC genotype & $(n=581)$ & $(n=651)$ \\
\hline Presence (+) & Presence (+) & $15(2.6)$ & $3(0.5)$ \\
\hline Presence (+) & Absence (-) & $37(6.4)$ & $19(2.9)$ \\
\hline Absence (-) & Presence $(+)$ & $142(24.4)$ & $142(21.7)$ \\
\hline
\end{tabular}

(c) Results for meta-analysis of the Japanese and Korean population

\begin{tabular}{|c|c|c|c|c|c|c|c|c|c|}
\hline \multicolumn{2}{|c|}{ Comparison } & \multicolumn{2}{|c|}{$\begin{array}{l}\text { Individual } \\
\text { association }\end{array}$} & \multicolumn{2}{|c|}{$\begin{array}{c}\text { Independent } \\
\text { association for } A\end{array}$} & \multicolumn{2}{|c|}{$\begin{array}{c}\text { Independent } \\
\text { association for } B\end{array}$} & \multirow{2}{*}{$\begin{array}{c}\text { Difference between } \\
\text { A-and } B \text {-association } \\
\text { Test }\{7\}\end{array}$} & \multirow{2}{*}{$\begin{array}{c}\begin{array}{c}\text { Combinatory } \\
\text { association }\end{array} \\
\text { Test }\{8\}\end{array}$} \\
\hline Factor A & Factor B & Test $\{1\}$ & Test $\{2\}$ & Test $\{3\}$ & Test $\{4\}$ & Test $\{5\}$ & Test $\{6\}$ & & \\
\hline BRAP & $9 p 21$ & $\mathrm{OR}_{\mathrm{A}}$ & $\mathrm{OR}_{\mathrm{B}}$ & ++ vs -+ & $\begin{array}{l}+- \text { vs }-- \\
\mathrm{OR}_{\mathrm{A}, \text { non- } \mathrm{B}}\end{array}$ & $++\mathrm{vs}+-$ & $\begin{array}{c}-+ \text { vs }-- \\
\text { OR }_{\text {non-A,B }}\end{array}$ & +- vs -+ & $\begin{array}{c}++\mathrm{vs}- \\
\mathrm{OR}_{\mathrm{A}, \mathrm{B}}\end{array}$ \\
\hline OR & & 2.01 & 1.42 & 2.31 & 1.90 & 1.87 & 1.39 & 1.35 & 3.33 \\
\hline$P$-value & & $1.3 \times 10^{-5}$ & 0.00096 & 0.018 & 0.0013 & NS & 0.0034 & NS & $2.4 \times 10^{-4}$ \\
\hline
\end{tabular}

Abbreviations: BRAP, BRCA1-associated protein; OR, odds ratio.

Patients and controls were stratified by the presence or absence of rs11066001 (BRAP)-GG genotype and rs1333049 (9p21)-CC genotype.

Factor $A$; rs 11066001-GG genotype, Factor B; rs1333049-CC genotype, test 1 and 2; association with factor $A$ and $B$, respectively,

$\mathrm{OR}_{\mathrm{A}}, \mathrm{OR}_{\mathrm{B}}, \mathrm{OR}_{\mathrm{A}, \text { non- } \mathrm{B}}, \mathrm{OR}_{\mathrm{non}-\mathrm{A}, \mathrm{B}}$, and $\mathrm{OR}_{\mathrm{A}, \mathrm{B}}$; odds ratios for individuals with factor $\mathrm{A}, \mathrm{B}, \mathrm{A}$ and non- $\mathrm{B}$, non- $\mathrm{A}$ and $\mathrm{B}$, and both $\mathrm{A}$ and $\mathrm{B}$, respectively.

$P, P$-values were corrected for multiple testing $(n=8)$. NS, not significant $(P \mathrm{C}>0.05)$.

In this study, we evaluated for the first time the associations of eight genetic variants with CAD in the Japanese and Korean populations. The power calculations for the study design (Supplementary Table 2) suggested that the sample sizes analyzed in this study were large enough to capture the associations, considering that more than $80 \%$ power at a significance level of $5 \%$ was desired for the negative metaanalysis. ${ }^{20}$ There are several other loci reported to be associated with $\mathrm{CAD}$ in European populations including those on chromosomes 2q33, 6q24, 12q24 and 21q22. ${ }^{10,11}$ We have not tested them in this study, because the minor allele frequency of each SNP was less than 5\% in the HapMap data for the Japanese (JPT) or Han Chinese (CHB) populations (Supplementary Table 1) and therefore the statistical power was too low to obtain a definite conclusion from our study design.

The association of the BRAP SNP with CAD was replicated in two East Asian populations, Japanese and Korean. Ozaki et al. ${ }^{12}$ initially found the association of the BRAP SNP with MI in one Japanese cohort and replicated the association in another Japanese cohort and Taiwanese subjects. It was suggested from a functional analysis that a higher expression of $B R A P$ might enhance the degree of inflammation through the activation of the NF- $\kappa \mathrm{B}$ protein and thereby had an important role in the pathogenesis of MI. ${ }^{12}$ The replicated association of BRAP with $\mathrm{CAD}$ in the Japanese and Korean populations in our study, especially in both the MI and AP groups, strongly suggested that rs11066001 was a reliable genetic risk factor for CAD in East Asians.

Considering the interaction between BRAP protein and Breast Cancer 1 protein $(B R C A 1),{ }^{21}$ there was a possibility that a significant association of $\mathrm{CAD}$ with $B R A P$ in the Korean population might be obtained because of a selection bias in the controls, because Korean cancer patients without CAD were included in the control panel in addition to the random healthy individuals. However, there was no significant difference in the minor allele frequencies of the BRAP SNP among patients with stomach cancer $(0.189, n=230)$, colon cancer (0.143, $n=147)$, lung cancer $(0.201, n=87)$ and hepatocellular carcinoma $(0.190, n=71)$, compared with the random healthy individuals (0.191, $n=178)$, suggesting that the BRAP SNP was a genetic risk factor for $\mathrm{CAD}$ and was not related to the cancer phenotype in the Korean population.

Another point to be addressed was that the Japanese controls were selected at random and not matched for age, gender and clinical backgrounds to the cases. The controls were especially younger than the cases and their average age was 39.0 years (Table 1 ), implying that the controls included the individuals who might develop CAD in future. Therefore, we divided the controls into two groups by age, more than 50 or less than 49 years, and compared the allele frequencies. It was found that the allele frequencies of all the tested SNPs were virtually identical in these two groups (data not shown) and that a significant association with $B R A P$ was found irrespective of the used controls. The minor allele frequencies of the BRAP SNPs were $0.344,0.251$ and 0.241 in the patients, older controls ( $57.3 \pm 10.6$ years old) and younger controls ( $34.6 \pm 5.8$ years old). ORs were 1.56 and 1.65 , whereas the $P c$ values were 0.0036 and $7.5 \times 10^{-9}$, respectively, when the older and younger controls were used. In addition, when the allele frequency data were compared between males and females in the 
controls, there was virtually no difference. These observations implied that the association of $B R A P$ with CAD in East Asians was relatively strong to be replicated even when the controls were not well matched to the cases in background data.

The other SNPs showed no significant association with CAD in the East Asian populations, in both allele distribution and genotype distribution, even when the cases were stratified by gender or by the presence of classical risk factors (data not shown). On the other hand, one SNP on chromosome 16q23 showed a marginal association with $\mathrm{CAD}$ in the Korean population $(\mathrm{OR}=0.69,95 \% \mathrm{CI}=0.55-0.88$, $P \mathrm{c}=0.02)$ but not in the Japanese population $(\mathrm{OR}=1.04,95 \%$ $\mathrm{CI}=0.86-1.26, P \mathrm{c}=\mathrm{ns}$ ) (Table 2). The observed association in the Korean population might be a false-positive finding, but a possibility remained that the association was specific to the the Korean population. Although the association in the Korean population was not significant when the $\mathrm{CAD}$ patients were stratified into $\mathrm{MI}(\mathrm{OR}=0.68$, $95 \% \mathrm{CI}=0.52-0.90, P \mathrm{c}=\mathrm{ns})$ and $\mathrm{AP}(\mathrm{OR}=0.71,95 \% \mathrm{CI}=0.53-0.95$, $P \mathrm{c}=\mathrm{ns}$ ), ORs showed a similar trend. The non-significant finding after the multiple tests might be because of the fact that the number of samples in each category was decreased. The association should be tested in future studies to investigate whether the contribution of the 16q23-linked susceptibility gene would be different between the Korean and Japanese populations depending on the additional genetic or environmental differences. Nevertheless, to explore the possible weak associations with the SNPs other than that in BRAP in East Asians, further replication studies are needed because our study design was not large enough to capture the weak association and our controls were not matched to the cases for the gender, age, sex and clinical backgrounds and might contain individuals who would develop CAD in future.

Finally, we found a synergistic effect of BRAP and 9p21 loci in susceptibility to CAD. As the association of CAD with the 9p21 locus was reported repeatedly in the European populations, ${ }^{6,7,22,23}$ it is of interest to investigate whether the association of $B R A P$ with $C A D$ and the synergistic interaction of BRAP with the 9p21 locus would be found in the Europeans.

In conclusion, rs11066001 in BRAP was associated with CAD in both Japanese and Korean populations. Our null findings of the other tested SNPs indicated the needs for extensive validation studies to apply the reported CAD-associated SNPs in the future risk assessment in East Asian populations, because the contribution of each diseaseassociated SNP might be different depending on the ethnic groups.

\section{ACKNOWLEDGEMENTS}

We thank Drs Megumi Takahashi, Shigeru Houda, and Michio Yasunami for their contributions in the initial course of the study. This work was supported in part by a Grant-in-Aid for Scientific Research from the Ministry of Education, Culture, Sports, Science and Technology of Japan, a grant for JapanKorea collaboration research from the Japan Society for the Promotion of Science, and a grant for Joint Research Project under the Korea-Japan Basic Scientific Cooperation Program from the Korea Science and Engineering Foundation.
1 Lopez, A. D., Mathers, C. D., Ezzati, M., Jamison, D. T. \& Murray, C. J. Global and regional burden of disease and risk factors, 2001: systematic analysis of population health data. Lancet 27, 1747-1757 (2006).

2 Wang, Q. Molecular genetics of coronary artery disease. Curr. Opin. Cardiol. 20, 182-188 (2005)

3 Ciruzzi, M., Schargrodsky, H., Rozlosnik, J., Pramparo, P., Delmonte, H., Rudich, V. et al. Frequency of family history of acute myocardial infarction in patients with acute myocardial infarction. Am. J. Cardiol. 80, 122-127 (1997).

4 Ozaki, K., Ohnishi, Y., lida, A., Sekine, A., Yamada, R., Tsunoda, T. et al. Functional SNPs in the lymphotoxin-alpha gene that are associated with susceptibility to myocardial infarction. Nat. Genet. 32, 650-654 (2002).

5 Wang, X., Ria, M., Kelmenson, P. M., Eriksson, P., Higgins, D. C., Samnegård, A. et al. Positional identification of TNFSF4, encoding OX40 ligand, as a gene that influences atherosclerosis susceptibility. Nat. Genet. 37, 365-372 (2005).

6 Wellcome Trust Case Control Consortium. Genome-wide association study of 14000 cases of seven common diseases and 3000 shared controls. Nature 447, 661-678 (2007).

7 Samani, N. J., Erdmann, J., Hall, A. S., Hengstenberg, C., Mangino, M., Mayer, B. et al. Genomewide association analysis of coronary artery disease. N. Engl. J. Med. 357, 443-453 (2007).

8 Erdmann, J., Grosshennig, A., Braund, P. S., König, I. R., Hengstenberg, C., Hall, A. S. et al. New susceptibility locus for coronary artery disease on chromosome 3q22.3. Nat. Genet. 41, 280-282 (2009).

9 Trégouët, D. A., König, I. R., Erdmann, J., Munteanu, A., Braund, P. S., Hall, A. S. et al. Genome-wide haplotype association study identifies the SLC22A3-LPAL2-LPA gene cluster as a risk locus for coronary artery disease. Nat. Genet. 41, 283-285 (2009).

10 Myocardial Infarction Genetics Consortium. Genome-wide association of early-onset myocardial infarction with single nucleotide polymorphisms and copy number variants. Nat. Genet 41, 334-341 (2009).

11 Gudbjartsson, D. F., Bjornsdottir, U. S., Halapi, E., Helgadottir, A., Sulem, P., Jonsdottir, G. M. et al. Sequence variants affecting eosinophil numbers associate with asthma and myocardial infarction. Nat. Genet. 41, 342-347 (2009).

12 Ozaki, K., Sato, H., Inoue, K., Tsunoda, T., Sakata, Y., Mizuno, H. et al. SNPs in BRAP associated with risk of myocardial infarction in Asian populations. Nat. Genet. 41, 329-333 (2009).

13 Morgan, T. M., Krumholz, H. M., Lifton, R. P. \& Spertus, J. A. Nonvalidation of reported genetic risk factors for acute coronary syndrome in a large-scale replication study. JAMA 297, 1551-1561 (2007).

14 Ozaki, K., Inoue, K., Sato, H., lida, A., Ohnishi, Y., Sekine, A. et al. Functional variation in LGALS2 confers risk of myocardial infarction and regulates lymphotoxin-alpha secretion in vitro. Nature 429, 72-75 (2004).

15 Hinohara, K., Nakajima, T., Takahashi, M., Houda, S., Sasaoka, T., Nakahara, K. et al. Replication of association between a chromosome 9p21 polymorphism with coronary artery disease in Japanese and Korean populations. J. Hum. Genet. 53, 357-359 (2008).

16 Kimura, A., Takahashi, M., Choi, B. Y., Bae, S. W., Hohta, S. et al. ack of association between LTA and LGALS2 polymorphisms and myocardial infarction in Japanese and Korean populations. Tissue Antigens 69, 265-269 (2007).

17 Hinohara, K., Nakajima, T., Sasaoka, T., Sawabe, M., Lee, B. S., Ban, J. et al. Replication studies for the association of PSMA6 polymorphism with coronary artery disease in East Asian populations. J. Hum. Genet. 54, 248-251 (2009).

18 Menashe, I., Rosenberg, P. S. \& Chen, B. E. PGA: Power calculator for case-control genetic association analyses. BMC Genetics 9, 36 (2008).

19 Svejgaard, A. \& Ryder, L. P. HLA and disease associations: detecting the strongest association. Tissue Antigens 43, 18-27 (1994).

20 Bertram, L., McQueen, M. B., Mullin, K., Blacker, D. \& Tanzi, R. E. Systematic metaanalyses of Alzheimer disease genetic association studies: the AlzGene database. Nat. Genet. 39, 17-23 (2007)

21 Li, S., Ku, C. Y., Farmer, A. A., Cong, Y. S., Chen, C. F. \& Lee, W. H. Identification of a novel cytoplasmic protein that specifically binds to nuclear localization signal motifs. J. Biol. Chem. 273, 6183-6189 (1998).

22 Helgadottir, A., Thorleifsson, G., Manolescu, A., Gretarsdottir, S., Blondal, T., Jonasdottir, A. et al. A common variant on chromosome 9p21 affects the risk of myocardial infarction. Science 316, 1491-1493 (2007).

23 McPherson, R., Pertsemlidis, A., Kavaslar, N., Stewart, A., Roberts, R., Cox, D. R. et al. A common allele on chromosome 9 associated with coronary heart disease. Science 316, 1488-1491 (2007).

Supplementary Information accompanies the paper on Journal of Human Genetics website (http://www.nature.com/jhg) 